\title{
A!
}

This is an electronic reprint of the original article.

This reprint may differ from the original in pagination and typographic detail.

Golubev, Dmitry S.; II'Ichev, Evgeni V.; Kuzmin, Leonid S.

\section{Single-Photon Detection with a Josephson Junction Coupled to a Resonator}

Published in:

Physical Review Applied

DOI:

10.1103/PhysRevApplied.16.014025

Published: 09/07/2021

Document Version

Publisher's PDF, also known as Version of record

Please cite the original version:

Golubev, D. S., Il'Ichev, E. V., \& Kuzmin, L. S. (2021). Single-Photon Detection with a Josephson Junction Coupled to a Resonator. Physical Review Applied, 16(1), [014025].

https://doi.org/10.1103/PhysRevApplied.16.014025

This material is protected by copyright and other intellectual property rights, and duplication or sale of all or part of any of the repository collections is not permitted, except that material may be duplicated by you for your research use or educational purposes in electronic or print form. You must obtain permission for any other use. Electronic or print copies may not be offered, whether for sale or otherwise to anyone who is not an authorised user. 


\title{
Single-Photon Detection with a Josephson Junction Coupled to a Resonator
}

\author{
Dmitry S. Golubev®, ${ }^{1,}{ }^{*}$ Evgeni V. Il'ichev $\circledast{ }^{2,3}$ and Leonid S. Kuzmin ${ }^{4,5}$ \\ ${ }^{1}$ Pico Group, QTF Centre of Excellence, Department of Applied Physics, Aalto University, P.O. Box 15100, \\ FI-00076 Aalto, Finland \\ ${ }^{2}$ Leibniz Institute of Photonic Technology, Albert-Einstein-Straße 9, 07745 Jena, Germany \\ ${ }^{3}$ Novosibirsk State Technical University, 20 Karl Marx Avenue, Novosibirsk, 630073, Russia \\ ${ }^{4}$ Chalmers University of Technology, 41296 Gothenburg, Sweden \\ ${ }^{5}$ Nizhny Novgorod State Technical University, Nizhny Novgorod, 603951, Russia
}

(Received 26 February 2021; revised 4 May 2021; accepted 15 June 2021; published 9 July 2021)

\begin{abstract}
We use a semiclassical formalism to optimize a microwave single-photon detector based on switching events of a current-biased Josephson junction coupled to a resonator. To detect very rare events, the average time between dark counts $\tau_{\text {dark }}$ should be maximized taking into account that the switching time $\tau_{\mathrm{sw}}$ should be sufficiently small. We demonstrate that these times can be tuned in a wide range by changing the junction parameters, and $\tau_{\text {dark }} / \tau_{\mathrm{sw}} \sim 10^{9}$ can be achieved. Therefore, a junction-resonator arrangement can be used to detect extremely low photon fluxes (e.g., for searching for galactic axions).
\end{abstract}

DOI: 10.1103/PhysRevApplied.16.014025

\section{INTRODUCTION}

The implementation of scalable superconducting solidstate systems offers promising perspectives for microwave quantum engineering [1-4]. In this context, effective single-photon detectors are required to properly manipulate weak microwave signals. This need is becoming crucial, in particular, for quantum communication $[5,6]$ and in the search for axions $[7,8]$ to test the consequences of the standard model of particle physics.

Several types of microwave single-photon detectors have been experimentally realized so far. One type is based on semiconducting quantum dots, in which photon absorption causes an electron jump from one quantum dot to another [9-11]. The efficiency of such detectors operated in the quantum coherent regime was theoretically analyzed in Ref. [12]. The detectors of the second type rely on superconducting qubits with a level spacing close to the photon energy [13-16]. Yet another detector type is based on a Josephson junction with strongly hysteretic current-voltage characteristics. The operation principle of this detector is simple - an absorbed photon switches the current-biased junction from the superconducting state to the resistive state, which results in a dc voltage signal. Some applications of this effect have already been demonstrated; see Refs. [17-19]. All types of single-photon detectors mentioned above have a narrow frequency band, which is necessary for capturing verylow-energy microwave photons. At present, broadband

*dmitry.golubev@aalto.fi detectors, such as transition-edge sensors or kinetic inductance detectors, are not sufficiently sensitive to resolve single photons in the microwave frequency range.

Here we theoretically analyze a particular type of Josephson-junction detector, which is assumed to operate at very low photon fluxes and should wait a long time for photon arrival. Accordingly, we require the detector to have the lowest possible dark-count rate. At the same time, the detector should very quickly switch to the resistive state after a detection event so as to avoid photon loss and to increase the detection efficiency. Such properties are required for the detection of very rare events such as decay of elementary particles. A natural figure of merit for this type of detector is the ratio of the average time between the dark counts, $\tau_{\text {dark }}$, and the switching time $\tau_{\text {sw }}$. For a good detector, one should require $\tau_{\text {dark }} / \tau_{\mathrm{sw}} \gg 1$. We demonstrate below that in a system with a junction coupled to a high-quality-factor resonator [see Fig. 1(a)] one can achieve $\tau_{\text {dark }} / \tau_{\text {sw }} \sim 10^{7}$ with typical parameters of the setup provided the superconducting leads of the junction, if made of aluminum, are cooled below $90 \mathrm{mK}$. In principle, one can even push this ratio to $10^{9}$. In the latter case, a junction having, for example, $\tau_{\mathrm{sw}}=1 \mu \mathrm{s}$ would have $\tau_{\text {dark }}=10^{3} \mathrm{~s}$. We show that while $\tau_{\mathrm{sw}}$ and $\tau_{\text {dark }}$ can be tuned in a wide range by changing the junction parameters, their ratio predominantly depends on the number of discreet energy levels in the Josephson potential well. A theoretical model of a similar detector was recently presented [20], where the minima of the two-dimensional potential of the junction-plus-resonator system were found, the splitting between the energy levels in the potential 

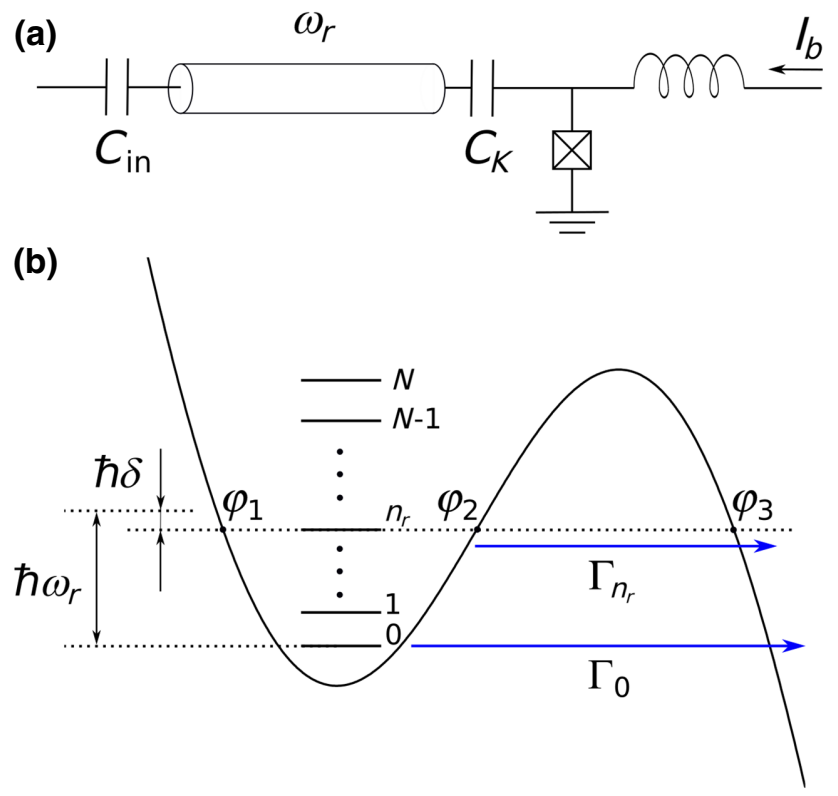

FIG. 1. (a) Josephson junction biased by the current $I_{b}$ and capacitively coupled to a resonator with frequency $\omega_{r}$. (b) Potential well of a tilted Josephson potential [Eq. (4)], which hosts $N+1$ discreet metastable energy levels with decay rates $\Gamma_{n}$, where $n=0,1, \ldots, N$. The frequency of the resonator is close to the transition frequency between levels 0 and $n_{r}$.

wells was determined, and the dark-count rate of the detector was roughly estimated as the switching rate of a weakly damped Josephson junction [21]. Here we extend the analysis in Ref. [20] in several ways. In particular, we include the transition-matrix elements between the energy levels in the model and solve the problem of the decay of metastable states localized in the potential wells in detail. In this way, we find not only the dark-count rate of the detector but also its switching time. We also analyze the effect of quasiparticles in the superconducting leads of the junction and losses in the resonator on the detector performance.

We briefly discuss the operating principle of the detector. A schematic is presented in Fig. 1(a). The detector consists of a conventional $\lambda / 2$ transmission-line resonator coupled to a grounded Josephson junction via a capacitor $C_{K}$ and to an input circuit via a capacitor $C_{\text {in }}$. An underdamped Josephson junction with strongly hysteric current-voltage characteristics is biased by the current $I_{b}$. This current can either be applied directly from the current source or be induced by magnetic flux via a superconducting loop attached to the junction. The potential well of tilted Josephson potential hosts $N+1$ energy levels $E_{n}$, where the index $n$ changes from 0 to $N$. By changing $I_{b}$, one can tune the system to a point where the condition $\hbar \omega_{r}=E_{n_{r}}-E_{0}+\hbar \delta$ is satisfied. Here $\delta \ll \omega_{r}$ is a small detuning, which may differ from zero at the optimal operating point, and $n_{r}$ is the number of the resonant level. Once a photon is created in the resonator, the level $n_{r}$ is populated after a short time inversely proportional to the coupling strength between this level and the resonator, which we denote as $g_{0 n_{r}}$. To ensure fast switching, we require the decay rate of this level, $\Gamma_{n_{r}}$, to be large. This implies that the energy $E_{n_{r}}$ should lie close to the top of the potential barrier. We find that preferably one should choose the second level from the top of the barrier with $n_{r}=N-1$ because at $n_{r}=N$ the dark-count rate is increased due to the large escape rate of the highest level. The total number of levels $N+1$ should be sufficiently large to make the dark-count time $\tau_{\text {dark }} \propto \exp (36 N / 5)$ as long as possible. On the other hand, $N$ cannot be too large so as to keep the coupling $g_{0 n_{r}}$ reasonably strong.

Below we present the theoretical model of the detector and discuss the trade-offs mentioned above in more detail. Our analysis is based on the theory of interlevel transitions in the Josephson potential well, which was proposed by Larkin and Ovchinnikov [22,23] and was further developed in Refs. [24-26]. We extend this theory by introducing the coupling between the junction and the resonator. The transitions between the energy levels of a tilted Josephson potential were detected experimentally by Martinis et al. [27] by measuring the changes in the switching rate of the junction under microwave irradiation. Later, the setup in Fig. 1 was used to implement a phase qubit, in which the two lowest energy levels in the well form the qubit and the higher levels are used for the readout $[28,29]$. Thus, the physics behind the proposed photon detector is well established and has been tested in experiments.

This paper is organized as follows: in Sec. II we introduce the model, in Sec. III we provide an approximate analytical solution, in Sec. IV we discuss how finite temperature, dissipation in the resonator, and nonequilibrium quasiparticles limit the performance of the detector, in Sec. $\mathrm{V}$ we present the results of a numerical simulation, and in Sec. VI we summarize the results.

\section{MODEL}

In this section we present the theoretical model of the system. In Sec. II A we provide the classical equations of motion, in Sec. II B we provide the corresponding quantum Hamiltonian, in Sec. II C we describe the semiclassical approximation, and in Sec. II D we briefly describe the numerical procedure. The derivation of the quantum Hamiltonian is presented in the Appendix.

\section{A. Classical equations of motion}

The classical dynamics of the system depicted in Fig. 1(a) is described by two coupled equations that can be derived from Kirchhoff's laws:

$$
\begin{aligned}
& \left(C+C_{K}\right) \frac{\hbar \ddot{\varphi}}{2 e}+I_{\mathrm{QP}}\left(\frac{\hbar \dot{\varphi}}{2}\right)+I_{c} \sin \varphi-C_{K} \dot{V}_{K}=I_{b}, \\
& \frac{2 Z_{0} C_{K} \omega_{r}^{3}}{\pi} \frac{\hbar \dot{\varphi}}{2 e}+\ddot{V}_{K}+\kappa_{r} \dot{V}_{K}+\omega_{r}^{2} V_{K}=0 .
\end{aligned}
$$


Here $C$ is the junction capacitance, $I_{\mathrm{QP}}(V)$ is the quasiparticle current through the junction, $I_{c}=\pi \Delta / 2 e R$ is the Josephson critical current [30], where $R$ is the normal-state resistance of the junction and $\Delta$ is the superconducting gap, $I_{b}$ is the bias current, $C_{K}$ is the coupling capacitor between the junction and the resonator, $Z_{0}$ is the characteristic impedance of the transmission-line resonator, and $V_{K}$ is the electric potential at the end of the resonator close to the capacitor $C_{K}$. The frequency of the fundamental mode of the resonator $\omega_{r}=\pi /\left(t_{0}+Z_{0} C_{K}\right)$, where $t_{0}$ is the flight time of a photon through the resonator. The damping rate of the resonator $\kappa_{r}$ is composed of the internal losses and the losses via the capacitor $C_{\text {in }}, \kappa_{r}=\kappa_{\text {int }}+(2 / \pi) \omega_{r}^{3} Z_{0}^{2} C_{\text {in }}^{2}$. The expressions in Eq. (1) are valid provided $\omega_{r} Z_{0} C_{K} \ll$ $\pi$ and the quality factor of the resonator is high, $Q_{r}=$ $\omega_{r} / \kappa_{r} \gg 1$.

\section{B. Quantum Hamiltonian}

In this and in the next section we ignore the dissipation in the resonator, setting $\kappa_{r}=0$, consider the zerotemperature limit, $T=0$, and ignore the effect of quasiparticles. These effects are discussed in Sec. IV. The system in Fig. 1(a) can be described by the quantum Hamiltonian (see the Appendix for the derivation)

$$
\hat{H}=\hat{H}_{J}+\hat{H}_{r}+\hat{H}_{\text {int }},
$$

where

$$
\hat{H}_{J}=-4 E_{C} \frac{\partial^{2}}{\partial \varphi^{2}}+U(\varphi)
$$

is the Hamiltonian of the junction, where $E_{C}=e^{2} / 2(C+$ $C_{K}$ ) is the charging energy and

$$
U(\varphi)=-E_{J} \cos \varphi-\frac{\hbar I_{b}}{2 e} \varphi+\frac{\hbar g^{2}}{\omega_{r}} \sqrt{\frac{E_{J}}{2 E_{C}}} \varphi^{2},
$$

is the Josephson potential tilted by the bias current $I_{b}$ with the quadratic correction coming from the coupling to the resonator, where $E_{J}=\hbar I_{c} / 2 e$ is the Josephson energy,

$$
\hat{H}_{r}=\hbar \omega_{r}\left(\hat{a}^{\dagger} \hat{a}+\frac{1}{2}\right)
$$

is the Hamiltonian of the resonator, and

$$
\hat{H}_{\mathrm{int}}=-i \hbar g\left(\frac{E_{J}}{2 E_{C}}\right)^{1 / 4}\left(\hat{a}^{\dagger}-\hat{a}\right) \varphi
$$

is the interaction term. Here

$$
g=\frac{C_{K} \omega_{r}^{2}}{\pi} \sqrt{\frac{Z_{0} R_{q}}{2}}\left(\frac{E_{C}}{8 E_{J}}\right)^{1 / 4}
$$

is the coupling strength between the resonator and the transition between levels 0 and 1 in the potential well of an unbiased junction (i.e., at $I_{b}=0$ ) and $R_{q}=h / e^{2}$ is the resistance quantum. Although Eq. (7) is specific for a coplanar resonator capacitively coupled to a junction, the Hamiltonian (2) is quite general and describes various types of resonators and couplings. The specifics of a particular setup affect only the expression for the coupling constant $g$. In addition, for certain types of coupling the combination $-i\left(\hat{a}^{\dagger}-\hat{a}\right)$ in the interaction term [Eq. (6)] should be replaced by the sum $\hat{a}^{\dagger}+\hat{a}$.

If one retains only the two lowest levels in a Josephson potential well with energies $E_{0}$ and $E_{1}$ and considers zero bias current, $I_{b}=0$, the phase operator can be approximated as $\varphi \rightarrow\left(2 E_{C} / E_{J}\right)^{1 / 4} \sigma_{x}$. Then the Hamiltonian (2) reduces to the usual Rabi Hamiltonian describing a transmon qubit [31]:

$$
\hat{H}_{\mathrm{Rabi}}=-\frac{E_{1}-E_{0}}{2} \sigma_{z}+\hbar \omega_{r}\left(\hat{a}^{\dagger} \hat{a}+\frac{1}{2}\right)-i \hbar g\left(\hat{a}^{\dagger}-\hat{a}\right) \sigma_{x} .
$$

\section{Semiclassical approximation}

In the limit $E_{J} \gg E_{C}$ the energy levels $E_{n}$ can be obtained from the Bohr-Sommerfeld quantization rule:

$$
\int_{\varphi_{1}}^{\varphi_{2}} d \varphi \sqrt{\frac{E_{n}-U(\varphi)}{4 E_{C}}}=\pi\left(n+\frac{1}{2}\right) .
$$

Here $\varphi_{1}$ and $\varphi_{2}$ are the classical turning points such that $U\left(\varphi_{1}\right)=E_{n}$ and $U\left(\varphi_{2}\right)=E_{n}$ [see Fig. 1(b)].

At finite bias current the levels become metastable. The corresponding decay rates can also be found from the semiclassical approximate expression

$$
\Gamma_{n}=\frac{\omega\left(E_{n}\right)}{2 \pi} \exp \left(-2 \int_{\varphi_{2}}^{\varphi_{3}} d \varphi \sqrt{\frac{U(\varphi)-E_{n}}{4 E_{C}}}\right) .
$$

Here $\omega\left(E_{n}\right)$ is the classical oscillation frequency in the potential well, which depends on the level energy $E_{n}$,

$$
\omega\left(E_{n}\right)=\frac{4 \pi}{\hbar}\left(\int_{\varphi_{1}}^{\varphi_{2}} \frac{d \varphi}{\sqrt{E_{C}\left[E_{n}-U(\varphi)\right]}}\right)^{-1},
$$

and $\varphi_{3}$ is the turning point on the other side of the potential barrier [see Fig. 1(b)].

For values of the bias current close to the critical current, $I_{c}-I_{b} \ll I_{c}$, one can approximately replace the potential in Eq. (4) by the cubic polynomial

$$
U=\frac{E_{J}}{2}\left(\alpha \phi^{2}-\frac{\phi^{3}}{3}\right),
$$

where $\phi=\varphi-\varphi_{\min }$ is the deviation of the phase from its equilibrium value $\varphi_{\min }$, at which the potential $U(\varphi)$ has a 
minimum, and

$$
\alpha=\sqrt{\frac{4 \pi \hbar g^{2}}{\omega_{r} \sqrt{8 E_{J} E_{C}}}+1-\frac{I_{b}^{2}}{I_{c}^{2}}} .
$$

We also need to know the matrix elements $\varphi_{m n}$ of the Josephson phase evaluated between the wave functions of levels $m$ and $n$. We again use the semiclassical approximation, which leads to the following expression:

$$
\begin{aligned}
\varphi_{m n}= & \frac{\omega\left(E_{m n}^{+}\right)}{2 \pi} \int_{-\pi / \omega\left(E_{m n}^{+}\right)}^{\pi / \omega\left(E_{m n}^{+}\right)} d t \varphi_{\mathrm{cl}}\left(t, E_{m n}^{+}\right) \\
& \sin \left[(m-n) \omega\left(E_{m n}^{+}\right) t\right] .
\end{aligned}
$$

Here $E_{m n}^{+}=\left(E_{m}+E_{n}\right) / 2$, and $\varphi_{\mathrm{cl}}$ is the solution of the classical equation of motion

$$
C \frac{\hbar \ddot{\varphi}_{\mathrm{cl}}}{2 e}+I_{c} \sin \varphi_{\mathrm{cl}}+\frac{2 \hbar g^{2}}{\omega_{r}} \sqrt{\frac{E_{J}}{2 E_{C}}} \varphi_{\mathrm{cl}}=I_{b},
$$

with the energy $E_{m n}^{+}$. Equation (14) can be solved analytically $[22,23]$ for $I_{c}-I_{b} \ll I_{c}$, where the cubic approximation [Eq. (12)] holds. In this case one finds

$$
\begin{aligned}
\varphi_{m n}= & \frac{\pi^{2} \sqrt{3}}{2}\left(\frac{12 U_{b}}{E_{J}}\right)^{1 / 3} \frac{\cos [(\pi / 6)-\theta]}{K^{2}(k)} \\
& \times \frac{(-1)^{|m-n|+1}(m-n)}{\sinh \left\{\pi(m-n)\left[K\left(\sqrt{1-k^{2}}\right) / K(k)\right]\right\}} .
\end{aligned}
$$

Here $U_{b}=2 E_{J} \alpha^{3 / 2} / 3$ is the height of the potential barrier, $K(k)$ is the complete elliptic integral,

$$
k=\sqrt{\frac{\sin \theta}{\cos [(\pi / 6)-\theta]}}
$$

is the ellipticity parameter, and

$$
\theta=\frac{2}{3} \arcsin \sqrt{\frac{E_{m n}^{+}-U\left(\varphi_{\min }\right)}{U_{b}}} .
$$

The semiclassical expressions (10) and (16) become invalid close to the top of the potential barrier, where one should use other approximations [23,24]. Here we avoid doing that so as to keep the model simple. Moreover, as mentioned above, the optimal choice for the resonant level is $n_{r}=N-1$. This level lies sufficiently far from the barrier top, where the approximation (16) is still applicable. For the same reason, we ignore the transition-matrix elements between the discreet levels and the continuous spectrum above the barrier top.

\section{Numerical solution of the problem}

Ideally, the dark-count rate and the rate of switching should be found by solving the time-evolution equation for the wave function of the system:

$$
i \hbar \frac{\partial \Psi_{k}(t, \varphi)}{\partial t}=\sum_{p=0}^{\infty} \hat{H}_{k p}(\varphi) \Psi_{p}(t, \varphi),
$$

where the indices $k$ and $p$ enumerate the number of photons in the resonator and $\hat{H}_{k p}(\varphi)$ is the sub-block of the Hamiltonian $\hat{H}$ relating the states with $k$ and $p$ photons. The initial wave function should be chosen such that the junction is initially in the ground state. The resonator should initially be in the ground state with zero photons if one is interested in the dark-count rate or it should host one photon if one wishes to find the switching rate. Having solved Eq. (19), one should evaluate the expression

$$
P(t)=\sum_{k=0}^{\infty} \int_{\varphi_{1}}^{\varphi_{2}}\left|\Psi_{k}(t, \varphi)\right|^{2},
$$

which determines the probability for the Josephson phase staying between the classical turning points $\varphi_{1}$ and $\varphi_{2}$ at time $t$ or, in other words, the probability of the switching event not occurring before the time $t$. Since the states in the potential well are metastable, the function (20) decays. Depending on the choice of the initial conditions, the timescale of this decay defines either the average time between the dark counts, $\tau_{\text {dark }}$, or the switching time $\tau_{\mathrm{sw}}$.

Since Eq. (19) is difficult to solve, we make the usual set of approximations. First, we expand the wave function of the system in the basis of the wave functions $\psi_{n}(\varphi)$ of the levels in the Josephson potential well.

$$
\Psi_{k}(t, \varphi)=\sum_{n=0}^{N} c_{n k}(t) \psi_{n}(\varphi)
$$

Then we add the imaginary part $-i \Gamma_{n} / 2$ to the energy of each discreet level, $E_{n} \rightarrow E_{n}-i \Gamma_{n} / 2$. The Hamiltonian $\hat{H}$ then acquires the form of a non-Hermitian matrix describing the decay of the initial metastable state in time. We assume that the coupling constant [Eq. (7)] is small, $g \ll \omega_{r}\left(2 E_{C} / E_{J}\right)^{1 / 4}$. This allows us to restrict the number of photons in the resonator to three values, $k=0,1,2$. Defining the coupling constants between the resonator and the interlevel transitions $m \leftrightarrow n$ as 


$$
g_{m n}=g\left(E_{J} / 2 E_{C}\right)^{1 / 4} \varphi_{m n}
$$

we write the Hamiltonian (2) in the form of a non-Hermitian $3(N+1) \times 3(N+1)$ matrix:

$$
\tilde{H}=\left(\begin{array}{ccc}
\left(E_{n}+\frac{\hbar \omega_{r}}{2}-i \frac{\hbar \Gamma_{n}}{2}\right) \delta_{m n} & i \hbar g_{m n} & 0 \\
-i \hbar g_{m n} & \left(E_{n}+\frac{3 \hbar \omega_{r}}{2}-i \frac{\hbar \Gamma_{n}}{2}\right) \delta_{m n} & i \sqrt{2} \hbar g_{m n} \\
0 & -i \sqrt{2} \hbar g_{m n} & \left(E_{n}+\frac{5 \hbar \omega_{r}}{2}-i \frac{\hbar \Gamma_{n}}{2}\right) \delta_{m n}
\end{array}\right) .
$$

The $(N+1) \times(N+1)$ sub-blocks on the diagonal of this matrix contain the complex energies of the metastable levels $E_{n}-i \Gamma_{n} / 2$. Namely, the sub-block in the top-left corner of the matrix describes the state with zero photons in the resonator, the sub-block in the middle describes the state with two photons, and the sub-block in the bottom-right corner describes the state with three photons. The off-diagonal sub-blocks originate from the interaction Hamiltonian (6) and contain the matrix elements (22). In this approximation, the probability (20) for the system to stay in the initial state $\Psi_{0}$ becomes

$$
P(t)=\left|e^{-\tilde{H} t} \Psi_{0}\right|^{2} .
$$

To find the dark-count rate, we choose $\Psi_{0}$ in the form of a $3(N+1)$-dimensional vector with all matrix elements equal to zero except for the first one, $\Psi_{0}^{T}=(1,0, \ldots, 0)$. The switching rate should be determined by setting all components of the initial wave function $\Psi_{0}$ to be zero except the component with number $N+2$, which should be equal to 1 . By our convention, this component corresponds to the ground state of the junction and one photon in the resonator.

\section{APPROXIMATE ANALYTICAL RESULTS}

Before proceeding to the numerical evaluation of the dark-count and switching rates, we present simple analytical approximations that may be useful for optimizing the detector parameters. We first consider the dark-count rate. In the lowest order of perturbation theory in the coupling strength $g$, the ground-state wave function is the product of the state with zero photons in the resonator, $k=0$, and of the ground state in the Josephson potential well with $n=0$. We denote this state as $|00\rangle$. Applying second-order perturbation theory in $g$ to the Hamiltonian $\tilde{H}$ (23), we find the corrected energy of this state in the form

$\tilde{E}_{00}=E_{0}-i \frac{\hbar \Gamma_{0}}{2}+\sum_{s=0}^{N} \frac{\hbar^{2} g_{0 s}^{2}}{E_{0}-E_{s}-\hbar \omega_{r}+i\left[\hbar\left(\Gamma_{s}+\Gamma_{0}\right) / 2\right]}$.

Taking the imaginary part of this expression, we find the dark-count rate of the device at zero temperature $\Gamma_{\text {dark }}$

$$
\begin{aligned}
& =\tau_{\text {dark }}^{-1}=-(2 / \hbar) \operatorname{Im} \tilde{E}_{00}: \\
& \Gamma_{\text {dark }}=\Gamma_{0}+\sum_{s=0}^{N} \frac{g_{0 s}^{2}\left(\Gamma_{s}+\Gamma_{0}\right)}{\left\{\left[\left(E_{s}-E_{0}\right) / \hbar\right]+\omega_{r}\right\}^{2}+\left[\left(\Gamma_{s}+\Gamma_{0}\right)^{2} / 4\right]} .
\end{aligned}
$$

The second term in this expression describes the increase of the dark-count rate due to the admixture of the excited states in the potential well caused by the interaction between the junction and the resonator. The approximate expression (26) can be used if $g \lesssim E_{1}-E_{0}$.

To find the switching rate we consider the state with one photon in the resonator $(k=1)$ and the ground state of the junction $(n=0)$, i.e., the state $|10\rangle$, and the state with zero photons in the resonator $(k=0)$ and the junction excited to the level $n_{r}$, i.e., the state $\left|0 n_{r}\right\rangle$. These two states are almost degenerate. We separate the two-dimensional subspace spanned by them and write the Hamiltonian (23) approximately as a $2 \times 2$ matrix:

$$
\tilde{H}^{\prime}=\left(\begin{array}{cc}
\tilde{E}_{0 n_{r}}+\frac{\hbar \omega_{r}}{2}-i \frac{\hbar \tilde{\Gamma}_{0 n_{r}}}{2} & -i g_{0 n_{r}} \\
i g_{0 n_{r}} & \tilde{E}_{10}+\frac{3 \hbar \omega_{r}}{2}-i \frac{\hbar \tilde{\Gamma}_{10}}{2}
\end{array}\right)
$$

Here the corrected energies and the decay rates are

$\tilde{E}_{0 n_{r}}=E_{n_{r}}+\sum_{s=1}^{N} \frac{\hbar^{2} g_{s n_{r}}^{2}\left(E_{n_{r}}-E_{s}-\hbar \omega_{r}\right)}{\left(E_{n_{r}}-E_{s}-\hbar \omega_{r}\right)^{2}+\left[\hbar^{2}\left(\Gamma_{s}+\Gamma_{n_{r}}\right)^{2} / 4\right]}$,

$$
\tilde{\Gamma}_{0 n_{r}}=\Gamma_{n_{r}}+\sum_{s=1}^{N} \frac{\hbar^{2} g_{s n_{r}}^{2}\left(\Gamma_{s}+\Gamma_{n_{r}}\right)}{\left(E_{n_{r}}-E_{s}-\hbar \omega_{r}\right)^{2}+\left[\hbar^{2}\left(\Gamma_{s}+\Gamma_{n_{r}}\right)^{2} / 4\right]}
$$

$$
\begin{aligned}
\tilde{E}_{10}= & E_{0}+\sum_{s \neq n_{r}} \frac{\hbar^{2} g_{0 s}^{2}\left(E_{0}+\hbar \omega_{r}-E_{s}\right)}{\left(E_{0}+\hbar \omega_{r}-E_{s}\right)^{2}+\left[\hbar^{2}\left(\Gamma_{s}+\Gamma_{0}\right)^{2} / 4\right]} \\
& +\sum_{s=0}^{N} \frac{2 \hbar^{2} g_{0 s}^{2}\left(E_{0}-E_{s}-\hbar \omega_{r}\right)}{\left(E_{0}-E_{s}-\hbar \omega_{r}\right)^{2}+\left[\hbar^{2}\left(\Gamma_{s}+\Gamma_{0}\right)^{2} / 4\right]}
\end{aligned}
$$




$$
\begin{aligned}
\tilde{\Gamma}_{10}= & \Gamma_{0}+\sum_{s \neq n_{r}} \frac{\hbar^{2} g_{0 s}^{2}\left(\Gamma_{s}+\Gamma_{0}\right)}{\left(E_{0}+\hbar \omega_{r}-E_{s}\right)^{2}+\left[\hbar^{2}\left(\Gamma_{s}+\Gamma_{0}\right)^{2} / 4\right]} \\
& +\sum_{s=0}^{N} \frac{2 \hbar^{2} g_{0 s}^{2}\left(\Gamma_{s}+\Gamma_{0}\right)}{\left(E_{0}-E_{s}-\hbar \omega_{r}\right)^{2}+\left[\hbar^{2}\left(\Gamma_{s}+\Gamma_{0}\right)^{2} / 4\right]}
\end{aligned}
$$

Note that the renormalized energies and the decay rates depend on the number of photons in the resonator.

After approximate reduction of the Hilbert space to two dimensions, the initial wave function $|10\rangle$ takes the form $\Psi_{0}^{T}=(0,1)$, and the probability (24) becomes

$$
\begin{aligned}
P(t) & \approx\left|e^{-i \tilde{H}^{\prime} t}\left(\begin{array}{c}
0 \\
1
\end{array}\right)\right|^{2} \\
& =e^{-\left[\left(\tilde{\Gamma}_{0 n_{r}}+\tilde{\Gamma}_{10}\right) t / 2\right]}\left|\cos \Omega t+i \frac{\sqrt{\Omega^{2}-g_{0 n_{r}}^{2}}}{\Omega} \sin \Omega t\right|^{2} .
\end{aligned}
$$

Here we have introduced the complex-valued "frequency"

$$
\Omega=\sqrt{\left(\frac{\delta \omega}{2}+i \frac{\tilde{\Gamma}_{0 n_{r}}-\tilde{\Gamma}_{10}}{4}\right)^{2}+g_{0 n_{r}}^{2}},
$$

where the detuning $\delta \omega$ is given by

$$
\hbar \delta \omega=\hbar \omega_{r}-\tilde{E}_{0 n r}+\tilde{E}_{10} .
$$

It differs from the detuning $\delta$, defined in Fig. 1(b), since the energies $\tilde{E}_{k n}$ are shifted relative to the bare energies $E_{n}$. The switching rate of the junction is given by the slowest decay rate of the function (32):

$$
\Gamma_{\mathrm{sw}}=\tau_{\mathrm{sw}}^{-1}=\frac{\tilde{\Gamma}_{0 n_{r}}+\tilde{\Gamma}_{10}}{2}-2|\operatorname{Im} \Omega|
$$

The approximate expression (35) is valid at sufficiently small detuning from resonance, $\delta \omega \lesssim \omega_{r} / n_{r}$, and for sufficiently weak coupling, $g \lesssim E_{1}-E_{0}$.

The switching rate [Eq. (35)] reaches its maximum value at resonance $\delta \omega=0$, where it becomes

$$
\Gamma_{\mathrm{sw}}^{\max }=\frac{\tilde{\Gamma}_{0 n_{r}}+\tilde{\Gamma}_{10}}{2}-2 \operatorname{Re}\left(\sqrt{\frac{\left(\tilde{\Gamma}_{0 n_{r}}-\tilde{\Gamma}_{10}\right)^{2}}{16}-g_{0 n_{r}}^{2}}\right) .
$$

Since the ground-state level is much stabler than the level $n_{r}$, one can usually omit the decay rate $\tilde{\Gamma}_{10}$ from Eq. (36). The maximum switching rate is limited by the slowest bottleneck process. Indeed, for strong coupling $\left|g_{0 n_{r}}\right|>$
$\tilde{\Gamma}_{0 n_{r}} / 4$ it is limited by the decay of the $n_{r}$ th energy level, $\Gamma_{\mathrm{sw}}^{\max }=\tilde{\Gamma}_{0 n_{r}} / 2$, while in the weak coupling limit, $\left|g_{0 n_{r}}\right| \ll$ $\tilde{\Gamma}_{0 n_{r}} / 4$, it becomes $\Gamma_{\mathrm{sW}}^{\max }=4 g_{0 n_{r}}^{2} / \tilde{\Gamma}_{0 n_{r}}$.

The dependence of the switching rate on the detuning [Eq. (35)] has the form of a peak with a rather unusual shape. It crosses over from a Lorentzian peak for weak coupling, $\left|g_{0 n_{r}}\right| \lesssim \tilde{\Gamma}_{0 n_{r}} / 4$, to a peak with a sharp cusp in the strong-coupling regime $\left|g_{0 n_{r}}\right| \gtrsim \tilde{\Gamma}_{0 n_{r}} / 4$; see Fig. 2(b). The half width of this peak can be found exactly:

$$
\begin{aligned}
& \delta \omega_{1 / 2}=\sqrt{\frac{16}{3} g_{0 n_{r}}^{2}-\frac{\tilde{\Gamma}_{0 n_{r}}^{2}}{4}}, \quad\left|g_{0 n_{r}}\right|>\frac{\tilde{\Gamma}_{0 n_{r}}}{4}, \\
& \delta \omega_{1 / 2}=2 F \sqrt{\frac{F^{2}-\left(g_{0 n_{r}}^{2} / 2\right)}{F^{2}+\left(g_{0 n_{r}}^{2} / 2\right)}}, \quad\left|g_{0 n_{r}}\right|<\frac{\tilde{\Gamma}_{0 n_{r}}}{4} .
\end{aligned}
$$

Here we have assumed that $\tilde{\Gamma}_{10} \ll \tilde{\Gamma}_{0 n_{r}}$, and introduced the combination

$$
F=\frac{\tilde{\Gamma}_{0 n_{r}}}{4}+\sqrt{\frac{\tilde{\Gamma}_{0 n_{r}}^{2}}{16}-g_{0 n_{r}}^{2}} .
$$

For very weak coupling $\left|g_{0 n_{r}}\right| \ll \tilde{\Gamma}_{0 n_{r}} / 4$ we find $\delta \omega_{1 / 2}=$ $\tilde{\Gamma}_{0 n_{r}}$, while in the opposite limit $\left|g_{0 n_{r}}\right| \gg \tilde{\Gamma}_{0 n_{r}} / 4$ we obtain $\delta \omega_{1 / 2}=2\left|g_{0 n_{r}}\right| / \sqrt{3}$.

Since the detuning $\delta \omega$ in our device is controlled by the bias current, it makes sense to convert the half width [Eq. (37)] into current units:

$$
\begin{aligned}
\Delta I_{b, 1 / 2} & =\left|\frac{\hbar \delta \omega_{1 / 2}}{\left[\partial\left(\tilde{E}_{n_{r}}-\tilde{E}_{0}\right) / \partial I_{b}\right]}\right| \\
& \approx \sqrt{\frac{2 E_{J}}{E_{C}}} \frac{I_{c}}{I_{b, r}}\left(1-\frac{I_{b, r}^{2}}{I_{c}^{2}}\right)^{3 / 4} \frac{e \delta \omega_{1 / 2}}{n_{r}} .
\end{aligned}
$$

Here $I_{b, r}$ is the value of the bias current at which the resonance condition $\tilde{E}_{n_{r}}-\tilde{E}_{0}=\hbar \omega_{r}$ is achieved.

We now estimate the maximum $\tau_{\text {dark }} / \tau_{\text {sw }}$ ratio. Assuming that the coupling is sufficiently strong,

$$
\left|g_{0 n_{r}}\right|>\tilde{\Gamma}_{0 n_{r}} / 4
$$

and the junction is tuned to resonance, we obtain

$$
\left.\frac{\tau_{\text {dark }}}{\tau_{\mathrm{sw}}}\right|_{\max }=\frac{\Gamma_{\mathrm{sw}}^{\max }}{\Gamma_{\text {dark }}}=\frac{\tilde{\Gamma}_{0 n_{r}}}{2 \Gamma_{\text {dark }}} .
$$


We can roughly estimate the rates $\Gamma_{\text {dark }}\left[\right.$ Eq. (26)] and $\tilde{\Gamma}_{0 n_{r}}$ [Eq. (29)] as

$$
\begin{aligned}
\Gamma_{\mathrm{dark}} & \approx \Gamma_{0}+\frac{g_{0 N}^{2} \Gamma_{N}}{(N+1)^{2} \omega_{r}^{2}}, \\
\tilde{\Gamma}_{0 n_{r}} & \approx \Gamma_{n_{r}}+\frac{g_{N n_{r}}^{2} \Gamma_{N}}{\left(N+1-n_{r}\right)^{2} \omega_{r}^{2}} .
\end{aligned}
$$

Since we have assumed the condition (40) to be satisfied, the second terms in these expressions tend to dominate. Hence, we obtain the following estimate valid for $n_{r}<N$ :

$$
\begin{aligned}
\left.\frac{\tau_{\mathrm{dark}}}{\tau_{\mathrm{sw}}}\right|_{\max } & \approx \frac{g_{N n_{r}}^{2}}{2 g_{0 N}^{2}}=\frac{\varphi_{N n_{r}}^{2}}{2 \varphi_{0 N}^{2}} \\
& \approx \frac{1}{2} \frac{\left(N-n_{r}\right)^{2}}{N^{2}} e^{2\left(\pi+2 c_{0}-\left[c_{0}\left(1+2 n_{r}\right) / N\right]\right) n_{r}} .
\end{aligned}
$$

Here we have introduced the constant

$$
c_{0}=\frac{\pi}{2 \sqrt{3}}\left(2 \frac{E(1 / \sqrt{2})}{K(1 / \sqrt{2})}-1\right)=0.4144 \ldots
$$

For a given number of levels in the well, the ratio (43) reaches the maximum value if one chooses $n_{r}=N-1$ (i.e., if one brings the energy level second closest to the barrier top in resonance with the resonator). In this case

$$
\left.\frac{\tau_{\text {dark }}}{\tau_{\mathrm{sw}}}\right|_{\max } \sim \frac{\exp \left\{2\left[\pi+\left(c_{0} / N\right)\right](N-1)\right\}}{2 N^{2}} .
$$

One can vary the absolute values of the $\tau_{\text {dark }}$ and $\tau_{\mathrm{sw}}$ by many orders of magnitude by changing the critical current, the bias current, or the charging energy of the junction. However, as Eq. (45) shows, $\tau_{\text {dark }} / \tau_{\text {sw }}$ predominantly depends on the number of the levels in the potential well at resonance irrespective of the specific values of $I_{c}, E_{C}$, or $I_{b}$.

\section{EFFECT OF QUASIPARTICLES AND DAMPING IN THE RESONATOR}

The dark-count rate and the efficiency of the detector depend on the temperature of the resonator environment, $T_{r}$, and on the temperature of the superconducting leads of the Josephson junction, $T_{S}$. First, we consider the latter effect. At finite $T_{S}$ the quasiparticles present in the leads cause up and down transitions between the neighboring energy levels of the Josephson potential well with rates $\Gamma_{\uparrow}^{\mathrm{QP}}$ and $\Gamma_{\downarrow}^{\mathrm{QP}}$, which satisfy the detailed-balance condition $k_{B} T_{S} \approx\left(E_{1}-E_{0}\right) \ln \left(\Gamma_{\downarrow}^{\mathrm{QP}} / \Gamma_{\uparrow}^{\mathrm{QP}}\right)$. Therefore, the levels with decay rates $\Gamma_{n}<\Gamma_{\downarrow}^{\mathrm{QP}}$ become thermally populated with the temperature $T_{S}$, and the dark-count rate increases.
To estimate this effect, we ignore the anharmonicity of the potential well and assume that the level splittings and quasiparticle transition rates are the same for all levels. We then obtain the temperature-dependent rate as

$$
\Gamma_{\text {dark }}\left(T_{S}\right) \approx \Gamma_{\text {dark }}+\Gamma_{\uparrow}^{\mathrm{QP}} W_{n_{0}} .
$$

Here $n_{0}$ is the level number such that $\Gamma_{n_{0}-1}<\Gamma_{\downarrow}^{\mathrm{QP}}<\Gamma_{n_{0}}$ and

$$
W_{n 0} \approx \frac{1-e^{-\left(E_{1}-E_{0}\right) / k_{B} T_{S}}}{e^{\left(E_{n_{0}}-E_{0}\right) / k_{B} T_{S}}-1}
$$

is the thermal population of this level obtained assuming the normalization condition $\sum_{n=0}^{n_{0}} W_{n}=1$. The latter condition follows from the observation that the level $n_{0}+1$ and higher ones are not populated because of their fast decay. The quasiparticle transition rates were derived in Ref. [32] and read

$$
\begin{aligned}
& \Gamma_{\uparrow}^{\mathrm{QP}}=\frac{\Gamma_{0}^{\mathrm{QP}}}{e^{\left(E_{1}-E_{0}\right) / k_{B} T_{S}}-1}, \quad \Gamma_{\downarrow}^{\mathrm{QP}}=\frac{\Gamma_{0}^{\mathrm{QP}}}{1-e^{-\left(E_{1}-E_{0}\right) / k_{B} T_{S}}}, \\
& \Gamma_{0}^{\mathrm{QP}}=\frac{1}{e^{2} R}\left|\left\langle 1\left|\sin \frac{\varphi}{2}\right| 0\right\rangle\right|^{2} \sqrt{\frac{2 \Delta}{E_{1}-E_{0}} \frac{n_{\mathrm{QP}}}{v_{0}} .}
\end{aligned}
$$

Here $n_{\mathrm{QP}}$ is the concentration of quasiparticles and $v_{0}$ is the density of states per unit spin in the superconductor. At low temperatures one finds $n_{\mathrm{QP}}=2 v_{0} \sqrt{2 \pi \Delta k_{B} T_{S}} e^{-\Delta / k_{B} T_{S}}$. It has been found experimentally that in aluminum, for example, it is very difficult to reduce $T_{S}$ below $120 \mathrm{mK}$ due to the presence of residual quasiparticles with the lowest reported concentration $n_{\mathrm{QP}} / 2 v_{0} \Delta \sim 10^{-9}$ [33,34]. Evaluating the matrix element of $\sin \varphi / 2$, we transform Eq. (48) to

$$
\begin{aligned}
\Gamma_{0}^{\mathrm{QP}}= & \frac{\Delta e^{-\left(\Delta / k_{B} T_{S}\right)}}{e^{2} R}\left(\frac{128 \pi^{2} k_{B}^{2} T_{S}^{2} E_{C}}{E_{J}^{3}}\right)^{1 / 4} \\
& \left(1+\frac{I_{c}}{\sqrt{I_{c}^{2}-I_{b}^{2}}}\right)
\end{aligned}
$$

Next we discuss the effect of unwanted thermal excitations in the resonator. For this purpose, we consider the time evolution of the occupation probabilities of the states $|10\rangle$ and $\left|0 n_{r}\right\rangle$ with one photon and zero photons in the resonator, which we denote as $p_{0}$ and $p_{1}$. For simplicity, we ignore quantum coherence and describe the system by two 
rate equations:

$$
\begin{aligned}
& \dot{p}_{0}=-\left(\Gamma_{\mathrm{dark}}+\Gamma_{\uparrow}^{\mathrm{QP}} W_{n_{0}}+\kappa_{r} N_{r}\right) p_{0}+\kappa_{r}\left(N_{r}+1\right) p_{1}, \\
& \dot{p}_{1}=\kappa_{r} N_{r} p_{0}-\left[\Gamma_{\mathrm{sw}}+\Gamma_{\downarrow}^{\mathrm{QP}}+\kappa_{r}\left(N_{r}+1\right)\right] p_{1} .
\end{aligned}
$$

Here $N_{r}=\left(e^{\hbar \omega_{r} / k_{B} T_{r}}-1\right)^{-1}$ is the Bose function containing the resonator temperature, $\kappa_{r} N_{r}$ is the rate of photon absorption by the resonator from its dissipative environment, and $\kappa_{r}\left(N_{r}+1\right)$ is the rate of spontaneous photon emission to the environment. From the expressions in Eq.
(50) one finds that the state with zero photons decays as $p_{0}(t) \propto \exp \left[-\Gamma_{\text {dark }}\left(T_{r}, T_{S}\right) t\right]$, where

$$
\Gamma_{\text {dark }}\left(T_{r}, T_{S}\right)=\Gamma_{\text {dark }}+\kappa_{r} N_{r}+\Gamma_{\uparrow}^{\mathrm{QP}} W_{n_{0}}
$$

is the total dark-count rate estimated in the limit $\Gamma_{\text {dark }}\left(T_{r}, T_{S}\right) \ll \Gamma_{\mathrm{sw}}$, and $\Gamma_{\text {dark }}$ is given by Eq. (26). Comparing the last two terms in Eq. (51) with the zerotemperature dark-count rate $\Gamma_{\text {dark }}$, we estimate the temperatures $T_{r}^{*}$ and $T_{S}^{*}$, below which the environment and the quasiparticle contributions can be ignored and the detector should demonstrate its best performance:

$$
\begin{aligned}
& T_{r}^{*}=\frac{\hbar \omega_{r}}{k_{B} \ln \left[1+\left(\kappa_{r} / \Gamma_{\text {dark }}\right)\right]}, \\
& T_{S}^{*} \approx \frac{\Delta+\left(n_{0}+1\right)\left(E_{1}-E_{0}\right)}{k_{B} \ln \left\{\left(\Delta / e^{2} \Gamma_{\text {dark }}\right) \sqrt{\left(8 \pi / R R_{q}\right)}\left(E_{C}^{1 / 4} / E_{J}^{1 / 4}\right)\left[1+\left(I_{c} / \sqrt{I_{c}^{2}-I_{b}^{2}}\right)\right]\right\}}
\end{aligned}
$$

Finally, we estimate the detector efficiency $\eta$, which can be obtained from the second expression in Eq. (50). Indeed, according to it, after absorption of a photon the probability $p_{1}$ decays in time as $p_{1}(t)=e^{-\left(\Gamma_{\mathrm{sw}}+\Gamma_{\downarrow}^{\mathrm{QP}}+\kappa_{r}\left(N_{T}+1\right)\right) t}$ until the photon is dissipated by the environment or by quasiparticles or the junction switches to the resistive state. The probability of the latter event determines the detector efficiency:

$$
\eta=\Gamma_{\mathrm{sw}} \int_{0}^{\infty} d t p_{1}(t)=\frac{\Gamma_{\mathrm{sw}}}{\Gamma_{\mathrm{sw}}+\Gamma_{\downarrow}^{\mathrm{QP}}+\kappa_{r}\left(N_{T}+1\right)} .
$$

As expected, the efficiency drops with the temperatures $T_{r}$ and $T_{S}$ and with the damping rate of the resonator $\kappa_{r}$.

\section{RESULTS OF NUMERICAL SIMULATION}

In this section we present the results of the numerical simulation described in Sec. II D and compare them with the simple approximations in Sec. III. We consider two sets of system parameters. First, we choose parameters typical for circuit-quantum-electrodynamics experiments. We then consider the parameter values at which the detector performance is significantly enhanced, but which may be more difficult to realize in experiments.

In Fig. 2(a) we plot $\tau_{\text {dark }}$ and $\tau_{\text {sw }}$ as functions of the bias current $I_{b}$ for the first set of parameters. In this simulation the system parameters are the normal-state resistance of the junction $R_{N}=500 \Omega$, the characteristic impedance of the transmission-line resonator $Z_{0}=50 \Omega$, the resonator frequency $\omega_{r} / 2 \pi=14.5 \mathrm{GHz}$, the junction capacitance $C=0.8 \mathrm{pF}$, and the coupling capacitance
$C_{K}=10 \mathrm{fF}$. Assuming that superconducting leads of the junction are made of aluminum with gap $\Delta=200 \mu \mathrm{eV}$, we find the critical current $I_{c}=\pi \Delta / 2 e R_{N} \approx 0.6 \mu \mathrm{A}$ and
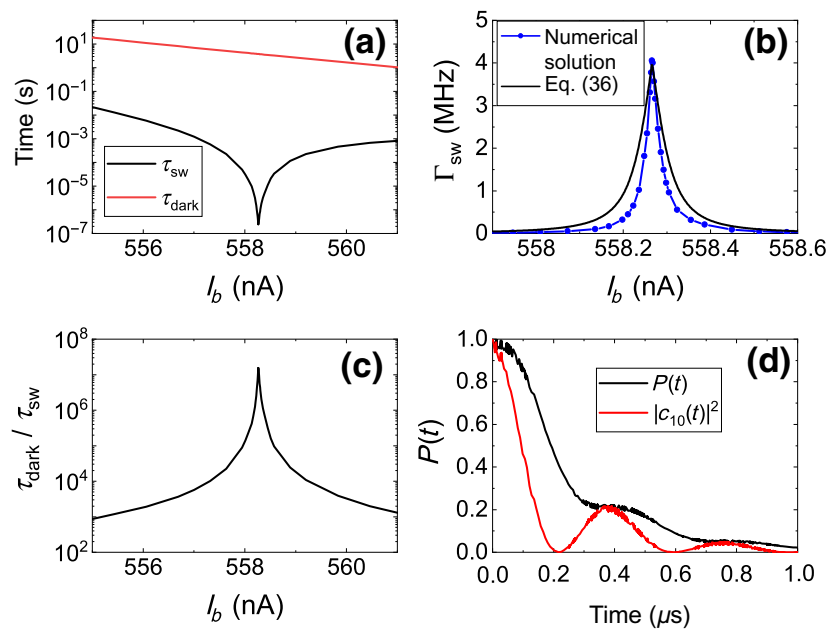

FIG. 2. (a) Dependence of the average time between dark counts $\left(\tau_{\text {dark }}\right.$; red line $)$ and of the switching time ( $\tau_{\text {sw }}$; black line) on the bias current $I_{b}$. (b) Bias dependence of the switching rate $\Gamma_{\mathrm{sw}}=\tau_{\mathrm{sw}}^{-1}$ in the vicinity of the bias current at which the resonance condition $\hbar \omega_{r}=\tilde{E}_{3}-\tilde{E}_{0}$ is met. The blue dots show the results of the numerical solution and the black line correspond to the approximate analytical expression [Eq. (35)] with the frequency converted to the current as in Eq. (39). (c) $\tau_{\text {dark }} / \tau_{\text {sw }}$ versus bias current. (d) Time dependence dependence of the probability $P(t)$ (black line), given by Eq. (24), and -the occupation probability $\left|c_{10}(t)\right|^{2}$ of the initial state $\psi_{0}=|10\rangle$ with one photon in the resonator and the ground state in the well of a tilted Josephson potential (red line). 
the Josephson energy $E_{J} / 2 \pi \hbar \approx 300 \mathrm{GHz}$. The charging energy of the junction $E_{C} / 2 \pi \hbar \approx 24.2 \mathrm{MHz}$, and hence we obtain the ratio $E_{J} / E_{C}=1.3 \times 10^{4}$. The McCumber parameter of a such junction is large, $\beta=2 e I_{c} R_{N}^{2}(C+$ $\left.C_{K}\right) / \hbar \approx 400$, which implies strongly hysteretic currentvoltage characteristics favorable for single-photon detection. The coupling constant between the junction and the resonator [Eq. (7)] is found to be $g / 2 \pi \approx 188.5 \mathrm{MHz}$. At bias current $I_{b} \approx 558 \mathrm{nA}$ the resonator frequency becomes equal to the transition frequency between levels 0 and $3, \omega_{r}=\left(\tilde{E}_{3}-\tilde{E}_{0}\right) / \hbar$. Thus, in this run of the numerical simulation we choose $n_{r}=3$. There are five levels in the potential well (i.e., $N=4$ ) in the whole interval of bias currents shown in Figs. 2(a)-2(c). At the bias current corresponding to resonance, the coupling constant for the $0 \leftrightarrow 3$ transition, defined in Eq. (22), takes the value $g_{03} / 2 \pi=1.5 \mathrm{MHz}$. We find the shortest switching time $\tau_{\mathrm{sw}}^{\min } \approx 0.25 \mu \mathrm{s}$, which is achieved at resonance, while the dark-count time $\tau_{\text {dark }}$ in this case becomes $3.8 \mathrm{~s}$. The minimum switching time obtained is comparable to dephasing times measured in good-quality phase qubits [35] $T_{2} \approx$ $0.3 \mu \mathrm{s}$, thus satisfying the condition $\tau_{\mathrm{sw}}^{\min }<T_{2}$, which is desirable for higher detection efficiency. We can estimate the quality factor of the resonator required for reliable photon detection as $Q_{r}>\omega_{r} \tau_{\mathrm{sw}}^{\min } \approx 2.2 \times 10^{4}$. For a lower quality factor a photon is dissipated in the resonator earlier than the junction switches and the detection efficiency [Eq. (54)] drops. For these parameters the dark-count rate is unaffected by the thermal population of the resonator for temperatures $T_{r} \lesssim T_{r}^{*} \approx 42 \mathrm{mK}$, where $T_{r}^{*}$ is defined in Eq. (52).

In Fig. 2(b) we plot the switching rate, $\Gamma_{\mathrm{sw}}=\tau_{\mathrm{sw}}^{-1}$, in the vicinity of the resonance with blue dots. For comparison, we also plot the analytical formula (35) with the black line. Both curves have the shape of a peak with a cusp. We use the following input parameters for the analytical model: the escape rate from the third level of an uncoupled junction [Eq. (10)], $\Gamma_{3}=7.2 \mathrm{MHz}$, the escape rate from the third level of an uncoupled junction increased by the coupling to the ground state of the resonator [Eq. (29)], $\tilde{\Gamma}_{03}=$ $8 \mathrm{MHz}$, and the escape rate for the state $|10\rangle$ [Eq. (31)], $\tilde{\Gamma}_{10}=1.6 \mathrm{~Hz}$. Since $g_{03}>\tilde{\Gamma}_{03} / 4$ and $\tilde{\Gamma}_{03} \gg \tilde{\Gamma}_{10}$, the maximum switching rate [Eq. (36)] is expected to be $\Gamma_{\mathrm{sw}}^{\max } \approx$ $\tilde{\Gamma}_{03} / 2=4 \mathrm{MHz}$, which perfectly agrees with the value obtained numerically. The half width of the peak in frequency units [Eq. (37)] $\delta \omega_{1 / 2}=21 \mathrm{MHz}$. This translates to the width of the current peak [Eq. (39)] $\Delta I_{b, 1 / 2}=63$ $\mathrm{pA}$, which is approximately 2 times bigger than the value obtained numerically, $\Delta I_{b, 1 / 2}=35 \mathrm{pA}$. The discrepancy between the analytical model and the numerical simulation comes from the rather simple approximation for the level splitting $\tilde{E}_{n_{r}}-\tilde{E}_{0} \approx n_{r} \sqrt{8 E_{J} E_{C} \alpha}$, which we use to derive the frequency-to-current conversion factor in Eq. (39). This approximation, however, is sufficiently accurate for a rough estimate of the current peak width. In Fig. 2(c) we plot $\tau_{\text {dark }} / \tau_{\mathrm{sw}}$, which characterizes the performance of the detector, as a function of the bias current. The maximum ratio is achieved at resonance, $\tau_{\text {dark }} / \tau_{\mathrm{sw}}=1.54 \times 10^{7}$. It is slightly higher than the estimate (45), which predicts $\tau_{\text {dark }} / \tau_{\mathrm{sw}}=9 \times 10^{6}$.

So far we have ignored the effect of quasiparticles in superconducting leads. Assuming the lowest effective temperature of the aluminum leads of the junction reported in qubit experiments, $T_{S}=120 \mathrm{mK}$, from Eqs. (48) and (49) we obtain $\Gamma_{\downarrow}^{\mathrm{QP}}=1.8 \mathrm{kHz}$. Next, we numerically find the decay rates of the first and second levels in the well, $\Gamma_{1} \approx$ $35 \mathrm{~Hz}$ anf $\Gamma_{2} \approx 22.6 \mathrm{kHz}$, and observe that $\Gamma_{1}<\Gamma_{10}^{\mathrm{QP}}<$ $\Gamma_{2}$. Thus, we set $n_{0}=2$ in Eq. (51), which results in darkcount rate $\Gamma_{\text {dark }}\left(0, T_{S}\right)=3 \mathrm{~Hz}$, dark-count time $\tau_{\text {dark }}=$ $0.36 \mathrm{~s}$, and $\tau_{\mathrm{dark}} / \tau_{\mathrm{sw}} \approx 1.4 \times 10^{6}$. Thus, the presence of nonequilibrium quasiparticles slightly reduces the darkcount time of the device. We find that one should cool the junction leads below the temperature [Eq. (53)] $T_{S}^{*}=110$ $\mathrm{mK}$ to achieve the dark-count time of $3.8 \mathrm{~s}$ reported above. Such temperatures may be easier to achieve in the proposed detector than in qubit devices because the leads of the junction, which are not electrically isolated superconducting islands, can be made sufficiently bulky. In addition, in our setup one can use normal-metal quasiparticle traps in a more straightforward manner.

In Fig. 2(d) we plot the time dependence of the probability $P(t)$ [Eq. (24)] at resonance (black line). We also show the occupation probability of the initial state $|10\rangle$, in which the resonator hosts one photon and the junction is in its ground state, which we denote as $\left|c_{10}(t)\right|^{2}$. This probability oscillates because of the coherent coupling between the resonator and the junction. The frequency of these oscillations is $\operatorname{Re} \Omega$, where $\Omega$ is defined in Eq. (33). For the system parameters given above, we find $\operatorname{Re} \Omega / 2 \pi=$ $1.42 \mathrm{MHz}$. Both functions shown in Fig. 2(d) also exhibit high-frequency small-amplitude oscillations, which are not captured by the analytical expression (32).

Next we consider another set of parameters and assume that level number 4 is aligned with the resonator (i.e., we choose $n_{r}=4$ ). We also choose $N=5$, which means there are six levels in the well. The parameters of the system are $R_{N}=7 \mathrm{k} \Omega, Z_{0}=50 \Omega, \omega_{r} / 2 \pi=14.5 \mathrm{GHz}$, $C=200 \mathrm{fF}$, and $C_{K}=5 \mathrm{fF}$. This results in critical current $I_{c} \approx 45 \mathrm{nA}$ and Josephson energy $E_{J} / 2 \pi \hbar \approx 22 \mathrm{GHz}$. The charging energy of the junction $E_{C} / 2 \pi \hbar \approx 97 \mathrm{MHz}$, and hence we obtain $E_{J} / E_{C}=230$. The McCumber parameter of such a junction is very high, $\beta=2 e I_{C} R_{N}^{2}\left(C+C_{K}\right) / \hbar \approx$ 1300 . With these parameters, the coupling constant [Eq. (7)] between the junction and the resonator $g / 2 \pi \approx 260$ MHz. At bias current $I_{b}=16.96 \mathrm{nA}$ the resonance condition $\hbar \omega_{r}=\tilde{E}_{4}-\tilde{E}_{0}$ is achieved. At this bias point the coupling constant for the $0 \leftrightarrow 4$ transition, given by Eq. (22), is rather small, $g_{04} / 2 \pi=84 \mathrm{kHz}$, which makes the experimental observation of the $0 \leftrightarrow 4$ transition difficult. In Fig. 3(a) we plot $\tau_{\text {dark }}$ and $\tau_{\mathrm{sw}}$ as functions of the 
(a)
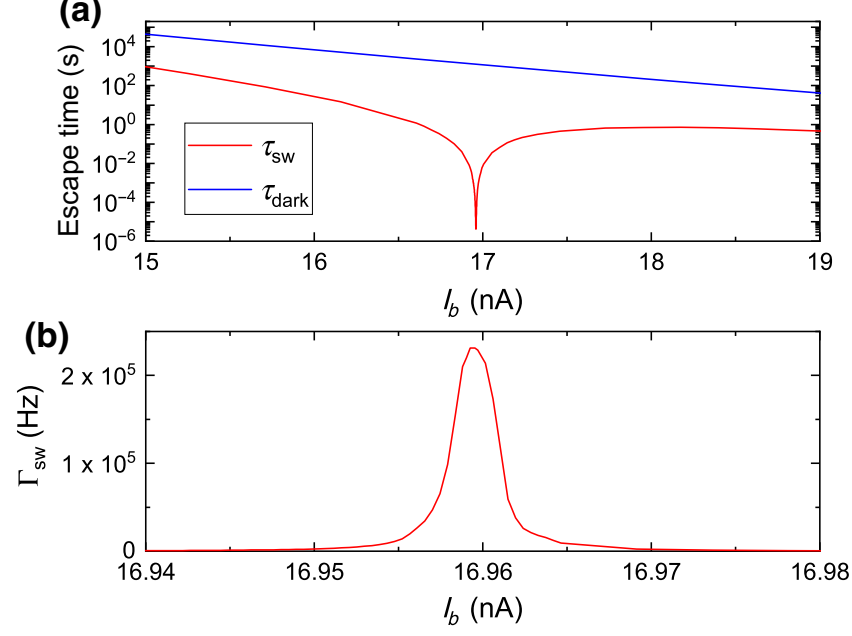

FIG. 3. (a) Dependence of the average time between dark counts $\left(\tau_{\text {dark }}\right.$; blue line $)$ and of the switching time $\left(\tau_{\mathrm{sw}}\right.$; red line) on the bias current $I_{b}$. (b) Dependence of the switching rate $\Gamma_{\mathrm{sw}}=\tau_{\mathrm{sw}}^{-1}$ on the bias current in the vicinity of $I_{b}=16.96 \mathrm{nA}$, at which the resonance condition $\hbar \omega_{r}=\tilde{E}_{4}-\tilde{E}_{0}$ occurs.

bias current $I_{b}$ for this set of parameters. We find the shortest switching time $\tau_{\mathrm{sw}}^{\min } \approx 4 \mu \mathrm{s}$, which is achieved at resonance, and dark-count time $\tau_{\text {dark }}=21 \mathrm{~min}$. The ratio between these two times is very large, $\tau_{\text {dark }} / \tau_{\mathrm{sw}}=$ $7.5 \times 10^{8}$. The estimate $(45)$ for this case predicts the ratio $4 \times 10^{9}$. As expected, by choosing $n_{r}=4$, we significantly increase $\tau_{\text {dark }} / \tau_{\mathrm{sw}}$ as compared with the previous set of parameters with $n_{r}=3$. The quality factor of the resonator required for reliable operation of the detector $Q_{r}>\omega_{r} \tau_{\mathrm{sw}}^{\min } \approx 4 \times 10^{5}$. With this quality factor the upper bound for the temperature [Eq. (52)] is $T_{r} \lesssim 30 \mathrm{mK}$.

We now discuss the effect of quasiparticles. Assuming again the effective temperature of the aluminum leads of the junction $T_{S}=120 \mathrm{mK}$, from Eqs. (48) and (49) we find $\Gamma_{\downarrow}^{\mathrm{QP}}=950 \mathrm{~Hz}$. Numerically we find $\Gamma_{2} \approx 7 \mathrm{~Hz}$ and $\Gamma_{3} \approx$ $3.7 \mathrm{kHz}$, which means $\Gamma_{2}<\Gamma_{10}^{\mathrm{QP}}<\Gamma_{3}$. Hence, we put $n_{0}=3$ in Eq. (51) and obtain dark-count rate $\Gamma_{\text {dark }}(T)=$ $1.2 \mathrm{~Hz}$, dark-count time $\tau_{\text {dark }}=0.8 \mathrm{~s}$, and $\tau_{\text {dark }} / \tau_{\mathrm{sw}} \approx 2 \times$ $10^{5}$. Thus, for this set of parameters the residual quasiparticles in the leads of the junction significantly degrade the performance of the detector. We find that to approach the zero-temperature value of $\tau_{\text {dark }}$, the junction leads should be cooled below $T_{S}^{*}=93 \mathrm{mK}$, which follows from Eq. (53).

In Fig. 3(b) we plot the switching rate in the vicinity of the resonance. It has the form of a narrow peak with maximum height $\Gamma_{\mathrm{sw}}^{\max } \approx 230 \mathrm{kHz}$ and half width $I_{b, 1 / 2} \approx 2.2 \mathrm{pA}$. The analytical model given by Eqs. (35), (37), and (39) with input parameters $\tilde{\Gamma}_{04}=1.1 \mathrm{MHz}$ and $\tilde{\Gamma}_{10}=1.3 \mathrm{mHz}$ predicts $\Gamma_{\mathrm{sw}}^{\max } \approx 575 \mathrm{kHz}, \delta \omega_{1 / 2} \approx 1.1$ $\mathrm{MHz}$, and $\Delta I_{b, 1 / 2} \approx 2.2 \mathrm{pA}$. In this case, the approximate model overestimates the maximum switching rate due to the slower nonexponential decay of the probability $P(t)$ at short times. On the other hand, in this case the analytical model very accurately predicts the width of the peak. This width is small, which makes practical realization of the detector with these parameters difficult.

The two examples considered above illustrate that one can push the $\tau_{\text {dark }} / \tau_{\mathrm{sw}}$ to very high values by increasing the number of the resonant level $n_{r}$. However, by doing so, one simultaneously decreases the width of the resonance peak in current units. One can partly compensate for that by choosing a larger coupling capacitor $C_{K}$ and in this way increasing the coupling constant $g$.

\section{CONCLUSION}

We propose and theoretically analyze a single-photon detector in the microwave frequency range, which consists of a current-biased Josephson junction coupled to a high-quality-factor resonator. We show that for typical system parameters, the ratio between the switching rate after arrival of a photon and the dark-count rate $\Gamma_{\mathrm{sw}} / \Gamma_{\text {dark }}$ can reach the order of $10^{7}$ provided the superconducting leads of the Josephson junction are cooled below $90 \mathrm{mK}$ and the environment of the resonator is cooled below $30 \mathrm{mK}$. With some effort, it should be possible to achieve even higher ratios on the order of $10^{9}$. Such a detector can operate at very low photon fluxes, where the time intervals between the photons may reach seconds or even hours. It can be useful in the detection of rare events such as the decay of elementary particles.

\section{ACKNOWLEDGMENTS}

This work was supported by the Academy of Finland Centre of Excellence program (Project No. 312057) and by the European Union's Horizon 2020 research and innovation programme under Grant Agreement No. 863313 (SUPERGALAX). It was also partly supported by the Ministry of Science and Higher Education of the Russian Federation (Grant No. FSUN-2020-0007) and by the Russian Science Foundation (Project No. 19-79-10170).

\section{APPENDIX: DERIVATION OF EQ. (2)}

In this section we derive the Hamiltonian (2). We follow the standard procedure, which has been used, for example, in Refs. [36,37] for slightly different systems. The classical equations (1), with dissipative terms omitted, can be derived from the Lagrangian

$$
\begin{aligned}
\mathcal{L}= & \frac{C+C_{K}}{2}\left(\frac{\hbar \dot{\varphi}}{2 e}\right)^{2}-E_{J}(1-\cos \varphi)+\frac{\hbar I_{b}}{2 e} \varphi \\
& +\frac{\pi\left(\dot{V}_{K}^{2}-\omega_{r}^{2} V_{K}^{2}\right)}{4 Z_{0} \omega_{r}^{3}}+C_{K} \frac{\hbar \varphi}{2 e} \dot{V}_{K} .
\end{aligned}
$$


We find the corresponding classical momenta

$$
\begin{aligned}
p_{\varphi} & =\frac{\partial \mathcal{L}}{\partial \dot{\varphi}}=\left(C+C_{K}\right) \frac{\hbar^{2} \dot{\varphi}}{4 e^{2}}, \\
p_{V_{k}} & =\frac{\partial \mathcal{L}}{\partial \dot{V}_{K}}=\frac{\pi \dot{V}_{K}}{2 Z_{0} \omega_{r}^{3}}+C_{K} \frac{\hbar \varphi}{2 e},
\end{aligned}
$$

and the classical Hamiltonian

$$
\begin{aligned}
H= & p_{\varphi} \dot{\varphi}+p_{V_{K}} \dot{V}_{K}-\mathcal{L} \\
= & \frac{4 e^{2}}{\hbar^{2}} \frac{p_{\varphi}^{2}}{2\left(C+C_{K}\right)}+E_{J}(1-\cos \varphi)-\frac{\hbar I_{b}}{2 e} \varphi \\
& +\frac{Z_{0} \omega_{r}^{3}}{\pi}\left(p_{V_{k}}-C_{K} \frac{\hbar \varphi}{2 e}\right)^{2}+\frac{\pi V_{K}^{2}}{4 Z_{0} \omega_{r}} .
\end{aligned}
$$

The quantum Hamiltonian (2) is obtained from the classical Hamiltonian (A3) by making the replacements

$$
\begin{aligned}
& p_{\varphi} \rightarrow-i \hbar \frac{\partial}{\partial \varphi}, \quad p_{V_{K}} \rightarrow i \sqrt{\frac{\pi \hbar \omega_{r}}{4 Z_{0} \omega_{r}^{3}}}\left(\hat{a}^{\dagger}-\hat{a}\right), \\
& V_{K} \rightarrow \sqrt{\frac{\hbar \omega_{r}^{2} Z_{0}}{\pi}}\left(\hat{a}^{\dagger}+\hat{a}\right),
\end{aligned}
$$

where $\hat{a}^{\dagger}$ and $\hat{a}$ are the creation and annihilation operators of the photons in the resonator.

[1] J. You and F. Nori, Atomic physics and quantum optics using superconducting circuits, Nature (London) 474, 589 (2011).

[2] G. Wendin, Quantum information processing with superconducting circuits: A review, Rep. Prog. Phys. 80, 106001 (2017).

[3] F. Arute, et al., Quantum supremacy using a programmable superconducting processor, Nature 574, 505 (2019).

[4] M. Grajcar, A. Izmalkov, and E. Il'ichev, Possible implementation of adiabatic quantum algorithm with superconducting flux qubits, Phys. Rev. B 71, 144501 (2005).

[5] N. Gisin and R. Thew, Quantum communication, Nat. Photonics 1, 165 (2007).

[6] S. Pogorzalek, K. G. Fedorov, M. Xu, A. Parra-Rodriguez, M. Sanz, M. Fischer, E. Xie1, K. Inomata, Y. Nakamura, E. Solano, A. Marx, F. Deppe, and R. Gross, Secure quantum remote state preparation of squeezed microwave states, Nat. Commun. 10, 2604 (2019).

[7] Leonid S. Kuzmin, Alexander S. Sobolev, Claudio Gatti, Daniele Di Gioacchino, Nicolo Crescini, Anna Gordeeva, and Evgeni Il'ichev, Single photon counter based on a Josephson junction at $14 \mathrm{GHz}$ for searching galactic axions, IEEE Appl. Supercond. 28, 2400505 (2018).

[8] T. Braine et al. (ADMX Collaboration), Extended Search for the Invisible Axion with the Axion Dark Matter Experiment, Phys. Rev. Lett. 124, 101303 (2020).
[9] O. Astafiev, S. Komiyama, T. Kutsuwa, V. Antonov, Y. Kawaguchi, and K. Hirakawa, Single-photon detector in the microwave range, Phys. Lett. 80, 4250 (2002).

[10] S. Gustavsson, M. Studer, R. Leturcq, T. Ihn, K. Ensslin, D. C. Driscoll, and A. C. Gossard, Frequency-Selective Single-Photon Detection Using a Double Quantum Dot, Phys. Rev. Lett. 99, 206804 (2007).

[11] W. Khan, P. P. Potts, S. Lehmann, C. Thelander, K. A. Dick, P. Samuelsson, and V. F. Maisi, Efficient and Continuous Microwave Photodetection in Hybrid CavitySemiconductor Nanowire Double Quantum Dot Diodes, arXiv:2011.05736

[12] C. H. Wong and M. G. Vavilov, Quantum efficiency of a single microwave photon detector based on a semiconductor double quantum dot, Phys. Rev. A 95, 012325 (2017).

[13] D. I. Schuster, A. A. Houck, J. A. Schreier, A. Wallraff, J. M. Gambetta, A. Blais, L. Frunzio, J. Majer, B. Johnson, M. H. Devoret, S. M. Girvin, and R. J. Schoelkopf, Resolving photon number states in a superconducting circuit, Nature 445, 515 (2007).

[14] B. R. Johnson, M. D. Reed, A. A. Houck, D. I. Schuster, Lev S. Bishop, E. Ginossar, J. M. Gambetta, L. DiCarlo, L. Frunzio, S. M. Girvin, and R. J. Schoelkopf, Quantum non-demolition detection of single microwave photons in a circuit, Nat. Phys. 6, 663 (2010).

[15] S. Kono, K. Koshino, Y. Tabuchi, A. Noguchi, and Y. Nakamura, Quantum non-demolition detection of an itinerant microwave photon, Nat. Phys. 14, 546 (2018).

[16] J.-C. Besse, S. Gasparinetti, M. C. Collodo, T. Walter, P. Kurpiers, M. Pechal, C. Eichler, and A. Wallraff, SingleShot Quantum Nondemolition Detection of Individual Itinerant Microwave Photons, Phys. Rev. X 8, 021003 (2018).

[17] Y.-F. Chen, D. Hover, S. Sendelbach, L. Maurer, S. T. Merkel, E. J. Pritchett, F. K. Wilhelm, and R. McDermott, Microwave Photon Counter Based on Josephson Junctions, Phys. Rev. Lett. 107, 217401 (2011).

[18] G. Oelsner, C. K. Andersen, M. Rehák, M. Schmelz, S. Anders, M. Grajcar, U. Hübner, K. Mølmer, and E. Il'ichev, Detection of Weak Microwave Fields with an Underdamped Josephson Junction, Phys. Rev. Appl. 7, 014012 (2017).

[19] G. Oelsner and E. Il'ichev, Switching dynamics of an underdamped Josephson junction coupled to a microwave cavity, Low Temp. Phys. 192, 169 (2018).

[20] D. V. Anghel, K. Kulikov, Y. M. Galperin, and L. S. Kuzmin, Electromagnetic radiation detectors based on Josephson junctions: Effective Hamiltonian, Phys. Rev. B 101, 024511 (2020).

[21] A. O. Caldeira and A. J. Leggett, Influence of Dissipation on Quantum Tunneling in Macroscopic Systems, Phys. Rev. Lett. 46, 211 (1981).

[22] A. I. Larkin and Yu N. Ovchinnikov, Current damping in superconducting junctions with nonequilibrium electron distribution functions, Sov. Phys. JETP 60, 1060 (1984).

[23] A. I. Larkin and Yu N. Ovchinnikov, Effect of level quantization on the lifetime of metastable states, Sov. Phys. JETP 64, 185 (1986).

[24] P. Kopietz and S. Chakravarty, Lifetime of metastable voltage states of superconducting tunnel junctions, Phys. Rev. B 38, 97 (1988). 
[25] K. S. Chow, D. A. Browne, and V. Ambegaokar, Quantum kinetics of a superconducting tunnel junction: Theory and comparison with experiment, Phys. Rev. B 37, 1624 (1988).

[26] Zhang Yun-Bo, Liang Jiu-Qing, and $\mathrm{Pu} \mathrm{Fu-Cho,}$ Path integral calculation of quantum tunneling for cubic potential at finite temperature, Phys. Sin. 7, 510 (1998).

[27] J. M. Martinis, M. H. Devoret, and J. Clarke, Energy-Level Quantization in the Zero-Voltage State of a Current-Biased Josephson Junction, Phys. Rev. Lett. 55, 1543 (1985).

[28] J. M. Martinis, S. Nam, J. Aumentado, and C. Urbina, Rabi Oscillations in a Large Josephson-Junction Qubit, Phys. Rev. Lett. 89, 117901 (2002).

[29] K. B. Cooper, Matthias Steffen, R. McDermott, R. W. Simmonds, Seongshik Oh, D. A. Hite, D. P. Pappas, and John M. Martinis, Observation of Quantum Oscillations between a Josephson Phase Qubit and a Microscopic Resonator Using Fast Readout, Phys. Rev. Lett. 93, 180401 (2004).

[30] V. Ambegaokar and A. Baratoff, Tunneling Between Superconductors, Phys. Rev. Lett. 10, 486 (1963).

[31] J. Koch, T. M. Yu, J. Gambetta, A. A. Houck, D. I. Schuster, J. Majer, A. Blais, M. H. Devoret, S. M. Girvin, and R. J. Schoelkopf, Charge-insensitive qubit design derived from the Cooper pair box, Phys. Rev. A 76, 042319 (2007).

[32] G. Catelani, J. Koch, L. Frunzio, R. J. Schoelkopf, M. H. Devoret, and L. I. Glazman, Quasiparticle Relaxation of
Superconducting Qubits in the Presence of Flux, Phys. Rev. Lett. 106, 077002 (2011).

[33] K. Serniak, S. Diamond, M. Hays, V. Fatemi, S. Shankar, L. Frunzio, R. J. Schoelkopf, and M. H. Devoret, Direct Dispersive Monitoring of Charge Parity in OffsetCharge-Sensitive Transmons, Phys. Rev. Appl. 12, 014052 (2019).

[34] E. T. Mannila, V. F. Maisi, and J. P. Pekola, A self-calibrating superconducting pair-breaking detector, ArXiv:2102.02254.

[35] Daniel Sank, R. Barends, Radoslaw C. Bialczak, Yu Chen, J. Kelly, M. Lenander, E. Lucero, Matteo Mariantoni, A. Megrant, M. Neeley, P. J. J. O’Malley, A. Vainsencher, H. Wang, J. Wenner, T. C. White, T. Yamamoto, Yi Yin, A. N. Cleland, and J. M. Martinis, Flux Noise Probed with Real Time Qubit Tomography in a Josephson Phase Qubit, Phys. Rev. Lett. 109, 067001 (2012).

[36] C. K. Andersen, G. Oelsner, E. Il'ichev, and K. Mølmer, Quantized resonator field coupled to a current-biased Josephson junction in circuit QED, Phys. Rev. A 89, 033853 (2014).

[37] J. Bourassa, J. M. Gambetta, A. A. Abdumalikov, O. Astafiev, Y. Nakamura, and A. Blais, Ultrastrong coupling regime of cavity QED with phase-biased flux qubits, Phys. Rev. A 80, 032109 (2009). 